\title{
DERIVED CATEGORY OF FIBRATIONS
}

\author{
L. Costa*, S. Di Rocco**, And R.M. Miró-Roig*
}

\begin{abstract}
In this paper we give a structure theorem for the derived category $D^{b}(X)$ of a Zariski locally trivial fibration $X$ over $Z$ with fiber $F$ provided both $F$ and $Z$ have a full strongly exceptional collection of line bundles.
\end{abstract}

\section{Introduction}

Let $X$ be a smooth projective variety defined over an algebraically closed field $K$ of characteristic zero and let $D^{b}(X)$ be the derived category of bounded complexes of coherent sheaves of $\mathcal{O}_{X}$-modules. $D^{b}(X)$ is one of the most important algebraic invariants of a smooth projective variety $X$ and, in spite of the increasing interest in understanding its structure, very little progress has been achieved. The study of $D^{b}(X)$ dates back to the late 70's when Beilinson described the derived category of projective spaces ([2]) and it has became an important topics in Algebraic Geometry. Among other reasons this is due to the Homological Mirror Symmetry Conjecture of Kontsevich [17] which states that there is an equivalence of categories between the derived category of coherent sheaves on a Calabi-Yau variety and the derived Fukai category of its mirror.

An important approach to determine the structure of $D^{b}(X)$ is to construct tilting bundles. Following terminology of representation theory (cf. [1]) a coherent sheaf $T$ of $\mathcal{O}_{X}$-modules on a smooth projective variety $X$ is called a tilting sheaf (or, when it is locally free, a tilting bundle) if

(i) it has no higher self-extensions, i.e. $\operatorname{Ext}_{X}^{i}(T, T)=0$ for all $i>0$,

(ii) the endomorphism algebra of $T, A=\operatorname{Hom}_{X}(T, T)$, has finite global homological dimension,

(iii) the direct summands of $T$ generate the bounded derived category $D^{b}\left(\mathcal{O}_{X}\right)$.

The importance of tilting sheaves relies on the fact that they can be characterized as those sheaves $T$ of $\mathcal{O}_{X}$-modules such that the functors $\mathbf{R H o m}_{X}(T,-): D^{b}(X) \longrightarrow$ $D^{b}(A)$ and $-\otimes_{A}^{\mathbf{L}} T: D^{b}(A) \longrightarrow D^{b}(X)$ define mutually inverse equivalences of the bounded derived categories of coherent sheaves on $X$ and of finitely generated right $A$ modules, respectively. The existence of tilting sheaves also plays an important role in the problem of characterizing the smooth projective varieties $X$ determined by their bounded derived category of coherent sheaves $D^{b}(X)$. For constructions of tilting

Received by the editors October 14, 2010.

1991 Mathematics Subject Classification. Primary 14F05.

* Partially supported by MTM2010-15256.

** Partially supported by Vetenskapsrådet's grant NT:2006-3539. 
bundles and their relations to derived categories we refer to the following papers: [1], [2], [3], [11] and [20].

In this paper we will focus our attention on the existence of tilting bundles which splits as a direct sum of line bundles on Zariski locally trivial fibrations. The search for tilting sheaves on a smooth projective variety $X$ splits naturally into two steps. First one has to find the so-called strongly exceptional collection of coherent sheaves on $X,\left(F_{0}, F_{1}, \ldots, F_{n}\right)$; then one has to show that $F_{0}, F_{1}, \ldots, F_{n}$ generate the bounded derived category $D^{b}(X)$.

Definition 1.1. A coherent sheaf $E$ on a smooth projective variety $X$ is called exceptional if it is simple and $\operatorname{Ext}_{\mathcal{O}_{X}}^{i}(E, E)=0$ for $i \neq 0$. An ordered collection $\left(E_{0}, E_{1}, \ldots, E_{m}\right)$ of coherent sheaves on $X$ is an exceptional collection if each sheaf $E_{i}$ is exceptional and $\operatorname{Ext}_{\mathcal{O}_{X}}^{i}\left(E_{k}, E_{j}\right)=0$ for $j<k$ and $i \geq 0$. An exceptional collection $\left(E_{0}, E_{1}, \ldots, E_{m}\right)$ is a strongly exceptional collection if in addition $\operatorname{Ext}_{\mathcal{O}_{X}}^{i}\left(E_{j}, E_{k}\right)=0$ for $i \geq 1$ and $j \leq k$. If an exceptional collection $\left(E_{0}, E_{1}, \ldots, E_{m}\right)$ of coherent sheaves on $X$ generates $D^{b}(X)$, then it is called full.

Thus each full strongly exceptional collection $\left(F_{0}, F_{1}, \ldots, F_{n}\right)$ defines a tilting sheaf $T=\oplus_{i=0}^{n} F_{i}$ and, vice versa, each tilting bundle whose direct summands are line bundles gives rise to a full strongly exceptional collection.

The now classical result of Beilinson [2] states that $\left(\mathcal{O}_{\mathbb{P}^{n}}, \mathcal{O}_{\mathbb{P}^{n}}(1), \ldots, \mathcal{O}_{\mathbb{P}^{n}}(n)\right)$ is a full strongly exceptional collection on $\mathbb{P}^{n}$. The following problem can be considered by now a natural and important question in Algebraic Geometry.

Problem 1.2. Characterize smooth projective varieties which have a full strongly exceptional collection and investigate whether there is one consisting of line bundles.

Note that not all smooth projective varieties have a full strongly exceptional collection of coherent sheaves. Indeed, the existence of a full strongly exceptional collection $\left(E_{0}, E_{1}, \ldots, E_{m}\right)$ of coherent sheaves on a smooth projective variety $X$ imposes a rather strong restriction on $X$, namely that the Grothendieck group $K_{0}(X)=K_{0}\left(\mathcal{O}_{X^{-}}\right.$ $\bmod )$ is isomorphic to $\mathbb{Z}^{m+1}$. Also notice that the above problem contains two subproblems. First to determine the existence of a full strongly exceptional collection on $X$ and then the existence of at least one consisting of line bundles. We want to stress that this second part is far from being redundant. There are several examples (for instance, the Grassmannians, see [14] and [15]) of varieties that have full strongly exceptional collections of vector bundles but that cannot have full strongly exceptional collections of line bundles. In [6], [7], [8] and [9], two of the authors constructed full strongly exceptional collections of line bundles on smooth toric varieties with a splitting fan and on smooth complete toric varieties with small Picard number. On [7] we constructed tilting bundles on projective bundles. The goal of this paper is to construct a full strongly exceptional collection consisting of line bundles for Zariski locally trivial fibrations. More precisely in the next section, after recalling the notion of a full strongly exceptional collection, we will show the following result.

Theorem 1.3. Let $\phi: X \rightarrow Z$ be a Zariski locally trivial fibration of smooth complex projective varieties with fiber $F$. Assume that $F$ and $Z$ have a full strongly exceptional 
collection of line bundles. Then, there is a full strongly exceptional collection of line bundles on $X$.

Two particular cases of this Theorem were proved by two of the authors in [6]. Indeed, in [6, Theorem 4.17], the case where $X$ is the trivial fibration over $Z$ with fiber $F$ (i.e. $X \cong Z \times F$ ) was proven, and in [7, Main Theorem], the case where $F \cong \mathbb{P}^{m}$ for some integer $m$ was covered.

We end the paper observing that for toric fibrations $\phi: X \rightarrow \mathbb{P}^{n}$ over $\mathbb{P}^{n}$ an explicit full strongly exceptional collection of line bundles can be provided.

\section{Derived category of Zariski locally trivial fibrations}

The goal of this section is to give a structure theorem for the derived category $D^{b}(X)$ of a Zariski locally trivial fibration $X$ over $Z$ with fiber $F$. This will be achieved constructing a full strongly exceptional collection of line bundles on $X$. We start by recalling the notions of exceptional sheaves, exceptional collections of sheaves, strongly exceptional collections of sheaves and full strongly exceptional collections of sheaves.

Definition 2.1. Let $X$ be a smooth projective variety.

(i) A coherent sheaf $E$ on $X$ is exceptional if $\operatorname{Hom}(E, E)=K$ and $\operatorname{Ext}_{\mathcal{O}_{X}}^{i}(E, E)=0$ for $i>0$,

(ii) An ordered collection $\left(E_{0}, E_{1}, \ldots, E_{m}\right)$ of coherent sheaves on $X$ is an exceptional collection if each sheaf $E_{i}$ is exceptional and $\operatorname{Ext}_{\mathcal{O}_{X}}^{i}\left(E_{k}, E_{j}\right)=0$ for $j<k$ and $i \geq 0$.

(iii) An exceptional collection $\left(E_{0}, E_{1}, \ldots, E_{m}\right)$ is a strongly exceptional collection if in addition $\operatorname{Ext}_{\mathcal{O}_{X}}^{i}\left(E_{j}, E_{k}\right)=0$ for $i \geq 1$ and $j \leq k$.

(iv) An ordered collection $\left(E_{0}, E_{1}, \ldots, E_{m}\right)$ of coherent sheaves on $X$ is a full (strongly) exceptional collection if it is a (strongly) exceptional collection and the coherent sheaves $E_{0}, E_{1}, \ldots, E_{m}$ generate the bounded derived category $D^{b}(X)$.

As an immediate consequence of the fact that for any smooth projective variety $X$ and for any line bundle $\mathcal{L}$ on $X$ to twist by $\mathcal{L}$ is an equivalence of the derived category $D^{b}(X)$, we have

Lemma 2.2. Let $X$ be a smooth projective variety and $\mathcal{M}$ a line bundle on $X$. If $\left(E_{0}, E_{1}, \ldots, E_{m}\right)$ is a full (strongly) exceptional collection of line bundles on $X$ then $\left(E_{0} \otimes \mathcal{M}, E_{1} \otimes \mathcal{M}, \ldots, E_{m} \otimes \mathcal{M}\right)$ is also a full (strongly) exceptional collection of line bundles on $X$.

Remark 2.3. As mentioned in the Introduction, the existence of a full strongly exceptional collection $\left(E_{0}, E_{1}, \ldots, E_{m}\right)$ of coherent sheaves on a smooth projective variety $X$ imposes a rather strong restriction on $X$, namely that the Grothendieck group $K_{0}(X)=K_{0}\left(\mathcal{O}_{X}\right.$-mod $)$ is isomorphic to $\mathbb{Z}^{m+1}$.

Let us illustrate the above definitions with examples:

Example 2.4. (1) The collection of line bundles $\left(\mathcal{O}_{\mathbb{P}^{n}}, \mathcal{O}_{\mathbb{P}^{n}}(1), \ldots, \mathcal{O}_{\mathbb{P}^{n}}(n)\right)$ is a full strongly exceptional collection of line bundles on $\mathbb{P}^{n}$. 
(2) Let $X_{1}$ and $X_{2}$ be two smooth projective varieties and let $\left(F_{0}^{i}, F_{1}^{i}, \ldots, F_{n_{i}}^{i}\right)$ be a full strongly exceptional collection of locally free sheaves on $X_{i}, i=1,2$. Then,

$$
\left(F_{0}^{1} \otimes F_{0}^{2}, F_{1}^{1} \otimes F_{0}^{2}, \ldots, F_{n_{1}}^{1} \otimes F_{0}^{2}, \ldots, F_{0}^{1} \otimes F_{n_{2}}^{2}, F_{1}^{1} \otimes F_{n_{2}}^{2}, \ldots, F_{n_{1}}^{1} \otimes F_{n_{2}}^{2}\right)
$$

is a full strongly exceptional collection of locally free sheaves on $X_{1} \times X_{2}$ (see [6]; Proposition 4.16). In particular, the collection of line bundles

$$
\begin{gathered}
\left(\mathcal{O}_{\mathbb{P}^{n}} \otimes \mathcal{O}_{\mathbb{P}^{m}}, \mathcal{O}_{\mathbb{P}^{n}}(1) \otimes \mathcal{O}_{\mathbb{P}^{m}}, \ldots, \mathcal{O}_{\mathbb{P}^{n}}(n) \otimes \mathcal{O}_{\mathbb{P}^{m}}, \ldots, \mathcal{O}_{\mathbb{P}^{n}} \otimes \mathcal{O}_{\mathbb{P}^{m}}(m),\right. \\
\left.\mathcal{O}_{\mathbb{P}^{n}}(1) \otimes \mathcal{O}_{\mathbb{P}^{m}}(m), \ldots, \mathcal{O}_{\mathbb{P}^{n}}(n) \otimes \mathcal{O}_{\mathbb{P}^{m}}(m)\right)
\end{gathered}
$$

is a full strongly exceptional collection of line bundles on $\mathbb{P}^{n} \times \mathbb{P}^{m}$.

(3) Let $X$ be a smooth complete variety which is the projectivization of a rank $r$ vector bundle $\mathcal{E}$ over a smooth complete variety $Z$ which has a full strongly exceptional collection of locally free sheaves. Then, $X$ also has a full strongly exceptional collection of locally free sheaves (See [6]; Proposition 4.9, [7]; Main Theorem).

As we said in the introduction $D^{b}(X)$ is an important algebraic invariant of a smooth projective variety but very little is known about the structure of $D^{b}(X)$. In particular, whether $D^{b}(X)$ is freely and finitely generated and, hence, we are lead to consider the following problem

Problem 2.5. Characterize smooth projective varieties $X$ which have a full strongly exceptional collection of coherent sheaves and, even more, investigate if there is one consisting of line bundles.

With full generality, this problem seems out of reach and only some particular cases have been addressed. For instance, in [16], Kawamata proved that the derived category of a smooth complete toric variety has a full exceptional collection of objects. In his collection the objects are sheaves rather than line bundles and the collection is only exceptional and not strongly exceptional. In the toric context, there are a lot of contributions to the above problem. For instance, it turns out that a full strongly exceptional collection made up of line bundles exists on projective spaces ([2]), products of projective spaces ([6]; Proposition 4.16), smooth complete toric varieties with Picard number $\leq 2$ ([6]; Corollary 4.13), smooth complete toric varieties with a splitting fan ([6]; Theorem 4.12) and some smooth complete toric varieties with Picard Number 3 ([18]). As mentioned in the introduction, not any variety has a full strongly exceptional collection made up of line bundles. For instance, in [12], Hille and Perling constructed an example of a smooth non Fano toric surface which does not have a full strongly exceptional collection made up of line bundles. Recently in [19] Michalek has also proved that for a big enough $n$, the blow up of $\mathbb{P}^{n}$ at two invariant points does not have strongly exceptional collection of line bundles. See also [10] for similar results on the Fano context.

Our goal is to describe the derived category of a Zariski locally trivial fibration. We will contribute to both parts of Problem 2.5 in the sense that we will address the problem of the existence of full strongly exceptional collections of coherent sheaves and, in our view more importantly, the existence of at least one consisting of line bundles. 
Remark 2.6. Let $\phi: X \rightarrow Z$ be a Zariski locally trivial fibration of smooth complex projective varieties with fiber $F$. Notice that any line bundle $\mathcal{L}$ on $F$ can be lifted to a line bundle on $X$. Indeed, let $D$ be a Weil divisor associated to $\mathcal{L}$. We can assume that $D$ is effective and by linearity we extend to any other Weil divisor. We consider the trivialization $U_{X}=U_{Z} \times F$ where $U_{X} \subset X$ and $U_{Z} \subset Z$ are Zariski open subsets and the divisor $D \times U_{Z}$ on $U_{X}$. Its closure in $X$ is a divisor $E$ on $X$ whose restriction to $F$ and to any fiber over $U_{Z}$ is $D$. By semicontinuity $\mathcal{O}_{X}(E)_{\mid F} \cong \mathcal{O}_{F}(D) \cong \mathcal{O}_{X}(E)_{\mid X_{z}}$ for any fiber $X_{z}$ of $\phi$.

Notation 2.7. Let $\phi: X \rightarrow Z$ be a Zariski locally trivial fibration of smooth complex projective varieties with fiber $F$. For any line bundle $\tilde{\mathcal{L}}$ on the fiber $F$ we will denote by $\mathcal{L}$ its lifting to $X$.

We are now ready to state the main result of this paper.

Theorem 2.8. Let $\phi: X \rightarrow Z$ be a Zariski locally trivial fibration of smooth complex projective varieties with fiber $F$. Let $\left(\tilde{\mathcal{L}_{1}}, \ldots, \tilde{\mathcal{L}_{u}}\right)$ be a full strongly exceptional collection of line bundles on $F$ and let $\left(\mathcal{E}_{1}, \ldots, \mathcal{E}_{v}\right)$ be a full strongly exceptional collection of line bundles on $Z$. Then, there is a line bundle $\mathcal{O}_{Z}(D)$ on $Z$ such that the ordered sequence

$$
\begin{gathered}
\left\{\left\{\phi^{*}\left(\mathcal{E}_{j} \otimes \mathcal{O}_{Z}(D)\right) \otimes \mathcal{L}_{1}\right\}_{1 \leq j \leq v},\left\{\phi^{*}\left(\mathcal{E}_{j} \otimes \mathcal{O}_{Z}(2 D)\right) \otimes \mathcal{L}_{2}\right\}_{1 \leq j \leq v}, \ldots\right. \\
\left.\ldots\left\{\phi^{*}\left(\mathcal{E}_{j} \otimes \mathcal{O}_{Z}(u D)\right) \otimes \mathcal{L}_{u}\right\}_{1 \leq j \leq v}\right\}
\end{gathered}
$$

is a full strongly exceptional sequence of line bundles on $X$.

Proof. It is a general fact that given a line bundle $\mathcal{L}$ on a Zariski locally trivial fibration on a smooth variety with fiber $F$ then $\left.\mathcal{L}\right|_{F}=\left.\mathcal{L}\right|_{X_{z}}$ for any fiber $X_{z}$. Therefore, $\left(\mathcal{L}_{1}, \ldots, \mathcal{L}_{u}\right)$ is a collection of line bundles on $X$ that reduces to a full strongly exceptional collection of line bundles on all the fibers.

We have to prove the existence of a line bundle $\mathcal{O}_{Z}(D)$ on $Z$ such that the above collection is full and satisfies the following cohomological conditions:

(a) $\operatorname{Ext}^{k}\left(\phi^{*}\left(\mathcal{E}_{i} \otimes \mathcal{O}_{Z}(j D)\right) \otimes \mathcal{L}_{j}, \phi^{*}\left(\mathcal{E}_{p} \otimes \mathcal{O}_{Z}(q D)\right) \otimes \mathcal{L}_{q}\right)=0$ for $k>0$ and $q \geq j$, and

(b) $\operatorname{Ext}^{k}\left(\phi^{*}\left(\mathcal{E}_{i} \otimes \mathcal{O}_{Z}(j D)\right) \otimes \mathcal{L}_{j}, \phi^{*}\left(\mathcal{E}_{p} \otimes \mathcal{O}_{Z}(q D)\right) \otimes \mathcal{L}_{q}\right)=0$ for $k \geq 0$ and $q<j$, which are equivalent to

$$
\begin{aligned}
& \left(a^{\prime}\right) \quad H^{k}\left(X, \phi^{*}\left(\mathcal{E}_{p} \otimes \mathcal{E}_{i}^{\vee} \otimes \mathcal{O}_{Z}((q-j) D)\right) \otimes \mathcal{L}_{q} \otimes \mathcal{L}_{j}^{\vee}\right)=0, \quad \text { for } k>0, \quad q \geq j \\
& \left(b^{\prime}\right) \quad H^{k}\left(X, \phi^{*}\left(\mathcal{E}_{p} \otimes \mathcal{E}_{i}^{\vee} \otimes \mathcal{O}_{Z}((q-j) D)\right) \otimes \mathcal{L}_{q} \otimes \mathcal{L}_{j}^{\vee}\right)=0, \quad \text { for } k \geq 0, \quad q<j .
\end{aligned}
$$

First of all recall that the Leray spectral sequence for $\phi$ gives us:

$$
\begin{aligned}
H^{r}\left(Z, R^{s} \phi_{*}\left(\phi ^ { * } \left(\mathcal{E}_{p} \otimes\right.\right.\right. & \left.\left.\left.\mathcal{E}_{i}^{\vee} \otimes \mathcal{O}_{Z}((q-j) D)\right) \otimes \mathcal{L}_{q} \otimes \mathcal{L}_{j}^{\vee}\right)\right) \\
& \Rightarrow H^{r+s}\left(X, \phi^{*}\left(\mathcal{E}_{p} \otimes \mathcal{E}_{i}^{\vee} \otimes \mathcal{O}_{Z}((q-j) D)\right) \otimes \mathcal{L}_{q} \otimes \mathcal{L}_{j}^{\vee}\right)
\end{aligned}
$$

and by the projection formula

$R^{s} \phi_{*}\left(\phi^{*}\left(\mathcal{E}_{p} \otimes \mathcal{E}_{i}^{\vee} \otimes \mathcal{O}_{Z}((q-j) D)\right) \otimes \mathcal{L}_{q} \otimes \mathcal{L}_{j}^{\vee}\right)=\mathcal{E}_{p} \otimes \mathcal{E}_{i}^{\vee} \otimes \mathcal{O}_{Z}((q-j) D) \otimes R^{s} \phi_{*}\left(\mathcal{L}_{q} \otimes \mathcal{L}_{j}^{\vee}\right)$. 
Thus,

$$
\begin{aligned}
H^{r}\left(Z, \mathcal{E}_{p} \otimes \mathcal{E}_{i}^{\vee} \otimes\right. & \left.\mathcal{O}_{Z}((q-j) D) \otimes R^{s} \phi_{*}\left(\mathcal{L}_{q} \otimes \mathcal{L}_{j}^{\vee}\right)\right) \\
& \Rightarrow H^{r+s}\left(X, \phi^{*}\left(\mathcal{E}_{p} \otimes \mathcal{E}_{i}^{\vee} \otimes \mathcal{O}_{Z}((q-j) D)\right) \otimes \mathcal{L}_{q} \otimes \mathcal{L}_{j}^{\vee}\right) .
\end{aligned}
$$

Claim: Since $\left(\mathcal{L}_{1}, \ldots, \mathcal{L}_{u}\right)$ is a collection of line bundles on $X$ that reduces to a full strongly exceptional collection of line bundles on all fibers $X_{z}, z \in Z$, for any $1 \leq j, q \leq u$ we have

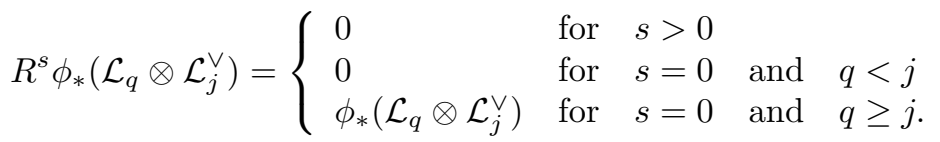

Proof of the Claim: ${ }^{1}$ Let $z \in Z$ be a point, and denote by $i_{z}$ the embedding of $z$ into $Z$. Since the morphism $\phi$ is smooth, by the base change,

$$
i_{z}^{*} R^{\bullet} \phi_{*}\left(\mathcal{L}_{q} \otimes \mathcal{L}_{j}^{\vee}\right)=\mathbb{H}^{\bullet}\left(X_{z}, \mathcal{L}_{q} \otimes \mathcal{L}_{j}^{\vee} \otimes \mathcal{O}_{X_{z}}\right)
$$

By the assumptions of the Claim, the latter graded vector space has the only nontrivial component in degree zero if $j<q$ and has no non-trivial component otherwise.

Assume there exist $l>0$ such that $R^{l} \phi_{*}\left(\mathcal{L}_{q} \otimes \mathcal{L}_{j}^{\vee}\right) \neq 0$. Let $d>0$ be the maximal positive integer among such $l$. Pick up a point $z$ in the support of the coherent sheaf $R^{l} \phi_{*}\left(\mathcal{L}_{q} \otimes \mathcal{L}_{j}^{\vee}\right)$, consider the spectral sequence that converges to $i_{z}^{*} R^{\bullet} \phi_{*}\left(\mathcal{L}_{q} \otimes \mathcal{L}_{j}^{\vee}\right)$ :

$$
E_{2}^{p, r}=L^{-p} i_{z}^{*} R^{r} \phi_{*}\left(\mathcal{L}_{q} \otimes \mathcal{L}_{j}^{\vee}\right) \Rightarrow L^{-p+r} i_{z}^{*} R^{\bullet} \phi_{*}\left(\mathcal{L}_{q} \otimes \mathcal{L}_{j}^{\vee}\right)
$$

and the term $E_{2}^{0, d}$. By assumption, it is a non-zero vector space. However, it survives in the above spectral sequence, since there are no differentials either going to or out of this term. Therefore, it gives a non-zero contribution to $L^{d} i_{z}^{*} R^{\bullet} \phi_{*}\left(\mathcal{L}_{q} \otimes \mathcal{L}_{j}^{\vee}\right)$ that is equal to zero by (5). Hence, this implies that $R^{s} \phi_{*}\left(\mathcal{L}_{q} \otimes \mathcal{L}_{j}^{\vee}\right)=0$ for all $s>0$. The case $q<j$ and $s=0$ follows step by step as this previous case.

In particular, $\left(b^{\prime}\right)$ follows from the Claim and (3). For the case $q=j,\left(a^{\prime}\right)$ follows from the Claim, $(3)$ and the fact that $\left(\mathcal{E}_{1}, \ldots, \mathcal{E}_{v}\right)$ is a strongly exceptional collection of line bundles on $Z$.

Finally assume that $q>j$. Since the line bundles $\mathcal{E}_{p}, 1 \leq p \leq v$ and $\mathcal{L}_{q}, 1 \leq q \leq u$ move in a finite set, there exists an ample line bundle $\mathcal{O}_{Z}(D)$ on $Z$ such that for any $r>0$ and $q-j>0$,

$$
H^{r}\left(Z, \mathcal{E}_{p} \otimes \mathcal{E}_{i}^{\vee} \otimes \mathcal{O}_{Z}((q-j) D) \otimes \phi_{*}\left(\mathcal{L}_{q} \otimes \mathcal{L}_{j}^{\vee}\right)\right)=0 .
$$

Once more by the Claim and (3) these vanishing ensures the remaining vanishing of $\left(a^{\prime}\right)$.

Putting altogether we get that indeed there exists an ample line bundle $\mathcal{O}_{Z}(D)$ on $Z$ such that the given ordered collection of line bundles is strongly exceptional.

Let us prove that it is full. To this end, observe that since by assumption $\left(\mathcal{E}_{1}, \ldots, \mathcal{E}_{v}\right)$ is a full strongly exceptional collection on $Z$, by Lemma 2.2 , for any $j>0$ we have

$$
D^{b}(Z)=\left\langle\mathcal{E}_{1} \otimes \mathcal{O}_{Z}(j D), \ldots, \mathcal{E}_{v} \otimes \mathcal{O}_{Z}(j D)\right\rangle .
$$

\footnotetext{
${ }^{1}$ See also the proof of Lemma 3.31 in [13].
} 
Therefore, it suffices to prove that

$$
D^{b}(X)=\left\langle\phi^{*} D^{b}(Z) \otimes \mathcal{L}_{1}, \ldots, \phi^{*} D^{b}(Z) \otimes \mathcal{L}_{u}\right\rangle
$$

where $\phi^{*} D^{b}(Z) \otimes \mathcal{L}_{k}$ denotes the full triangulated subcategory in $D^{b}(X)$ generated by the objects $\left\{\phi^{*}\left(\mathcal{E}_{j}\right) \otimes \mathcal{L}_{k}\right\}_{1 \leq j \leq v}$.

To this end, it is enough to check that $\left\langle\phi^{*} D^{b}(Z) \otimes \mathcal{L}_{1}, \ldots, \phi^{*} D^{b}(Z) \otimes \mathcal{L}_{u}\right\rangle$ contains all the objects $\mathcal{O}_{x}, x \in X$ since the set $\left\{\mathcal{O}_{x} \mid x \in X\right\}$ is a spanning class for $D^{b}(X)$ (See [13]; Proposition 3.17). Since every point $x \in X$ belongs to some fiber $X_{z}:=\phi^{-1}(z)$, $z \in Z$, and we have already seen that $\left(\mathcal{L}_{1}, \ldots, \mathcal{L}_{u}\right)$ reduces to a collection $\left(\tilde{\mathcal{L}}_{1}, \ldots, \tilde{\mathcal{L}_{u}}\right)$ that generates $D^{b}\left(X_{z}\right)$, we get

$$
\mathcal{O}_{x} \in\left\langle\tilde{\mathcal{L}_{1}}, \ldots, \tilde{\mathcal{L}_{u}}\right\rangle
$$

So, since $\phi^{*}\left(\mathcal{O}_{z}\right)=\mathcal{O}_{X_{z}}$, the sheaf $\mathcal{O}_{x}$ belongs to

$$
\left\langle\phi^{*} D^{b}(Z) \otimes \mathcal{L}_{1}, \ldots, \phi^{*} D^{b}(Z) \otimes \mathcal{L}_{u}\right\rangle .
$$

Putting altogether we get that

$$
\begin{gathered}
\left\{\left\{\phi^{*}\left(\mathcal{E}_{j} \otimes \mathcal{O}_{Z}(D)\right) \otimes \mathcal{L}_{1}\right\}_{1 \leq j \leq v},\left\{\phi^{*}\left(\mathcal{E}_{j} \otimes \mathcal{O}_{Z}(2 D)\right) \otimes \mathcal{L}_{2}\right\}_{1 \leq j \leq v}, \ldots\right. \\
\left.\ldots\left\{\phi^{*}\left(\mathcal{E}_{j} \otimes \mathcal{O}_{Z}(u D)\right) \otimes \mathcal{L}_{u}\right\}_{1 \leq j \leq v}\right\}
\end{gathered}
$$

generates $D^{b}(X)$.

Remark 2.9. As a consequence of [21]; Theorem 2, Samokhin obtained the existence of a full exceptional collection of coherent sheaves on flat fibrations. Nevertheless, in general, the collection given in [21] is not strongly exceptional.

Remark 2.10. Moreover we observe that for toric fibrations over $\mathbb{P}^{n}$ a stronger result is true. Indeed, consider $\phi: X \rightarrow \mathbb{P}^{n}$ a toric $F$-fibration over $\mathbb{P}^{n}$. Let $\left(\tilde{\mathcal{L}_{1}}, \ldots, \tilde{\mathcal{L}_{u}}\right)$ be a full strongly exceptional collection of line bundles on $F$ and take on $\mathbb{P}^{n}$ the usual full strongly exceptional collection of line bundles $\left(\mathcal{O}_{\mathbb{P}^{n}}, \ldots, \mathcal{O}_{\mathbb{P}^{n}}(n)\right)$. Then in this particular case, following the same ideas as in the proof of Theorem 2.8 and strongly using the fact that we have an explicit control of the cohomology of line bundles on this toric fibration, we get that the ordered sequence

$$
\left\{\left\{\phi^{*}\left(\mathcal{O}_{\mathbb{P}^{n}}\right) \otimes \mathcal{L}_{1}\right\}_{1 \leq j \leq v},\left\{\phi^{*}\left(\mathcal{O}_{\mathbb{P}^{n}}(1)\right) \otimes \mathcal{L}_{2}\right\}_{1 \leq j \leq v}, \ldots,\left\{\phi^{*}\left(\mathcal{O}_{\mathbb{P}^{n}}(n)\right) \otimes \mathcal{L}_{u}\right\}_{1 \leq j \leq v}\right\}
$$

is a full strongly exceptional sequence of line bundles on $X$.

\section{Acknowledgements}

The authors thank the referee for his/her useful comments which have improved considerably our work. We also thank A. Samokhin for helpful comments. This work was initiated during a visit of the second author at the University of Barcelona, which she thanks for its hospitality. 


\section{References}

[1] D. Baer, Tilting sheaves, Manusc. Math. 60 (1988), 323a-347.

[2] A.A. Beilinson, Coherent sheaves on $\mathbb{P}^{n}$ and Problems of Linear Algebra, Funkt. Anal. Appl. 12 (1979), 214-216.

[3] A.I. Bondal, Representation of associative algebras and coherent sheaves, Math. USSR Izvestiya 34 (1990), 23-42.

[4] A. Bondal and D. Orlov, Semiorthogonal decomposition for algebraic varieties, preprint:math.AG/9506012.

[5] L. Borisov, Z. Hua, On the conjecure of King for smmoth toric Deligne-Mumford stacks, Adv. Math. 221 (2009) 1, 277-301.

[6] L. Costa and R.M. Miró-Roig, Tilting sheaves on toric varieties, Math. Z. 248 (2004), 849-865.

[7] L. Costa and R.M. Miró-Roig, Derived categories of projective bundles, Proc. Amer. Math. Soc. 133 (2005), 2533-2537.

[8] L. Costa and R.M. Miró-Roig, Derived category of Toric varieties with small Picard number, Preprint 2008

[9] L. Costa and R.M. Miró-Roig, Frobenius splitting and Derived category of Toric varieties, To appear Illinois Journal of Mathematics.

[10] A. Efimov, Maximal lengths of exceptional collections of line bundles , arXiv:1010.3755 , 2010.

[11] A.N. Rudakov et al. Helices and vector bundles: Seminaire Rudakov Lecture Note Series, 148 (1990) Cambridge University Press.

[12] L. Hille, M. Perling, A counterexample to King's conjecture, Compos. Math. 142 (2006), 15071521.

[13] D. Huybrechts, Fourier-Mukai Transforms in Algebraic Geometry, Oxford Mathematical Monographs (2006).

[14] M. M. Kapranov, On the derived category of coherent sheaves on Grassmann manifolds, Math. USSR Izvestiya, 24 (1985), 183-192.

[15] M. M. Kapranov, On the derived category of coherent sheaves on some homogeneous spaces, Invent. Math., 92 (1988), 479-508.

[16] Y. Kawamata, Derived categories of toric varieties, Michigan Math. J. 54 (2006), 517-535.

[17] M. Kontsevich, Homological algebra of mirror symmetry, Proceedings of the International Congress of Mathematicians, Vol. 1, 2 (1994), 120-139, Birkhäuser, Basel, 1995.

[18] M. Lason, M. Michalek, On the full, strongly exceptional collections on toric varieties with Picard number three, arXiv:1003.2047, 2010.

[19] M. Michalek, Family of counterexamples to King's conjecture, Preprint 2010.

[20] D.O. Orlov, Projective bundles, monoidal transformations, and derived categories of coherent sheaves, Math. USSR Izv. 38 (1993), 133-141.

[21] A. Samokhin, Some remarks on the derived categories of coherent sheaves on homogeneous spaces J. London Math. Soc. (2) 76 (2007), 122134.

Facultat de Matemàtiques, Departament d’Algebra i Geometria, Gran Via de les Corts Catalanes 585, 08007 Barcelona, SPAiN

E-mail address: costa@ub.edu

Department of Mathematics, KTH, SE-10044 Stockholm, Sweden

E-mail address: dirocco@math.kth.se

Facultat de Matemàtiques, Departament d'Algebra i Geometria, Gran Via de les Corts Catalanes 585, 08007 Barcelona, SPAIN

E-mail address: miro@ub.edu 